FUNCTION SPACES XII

BANACH CENTER PUBLICATIONS, VOLUME 119

INSTITUTE OF MATHEMATICS

POLISH ACADEMY OF SCIENCES

WARSZAWA 2019

\title{
ON COMPACTNESS THEOREMS FOR LOGARITHMIC INTERPOLATION METHODS
}

\author{
BLANCA F. BESOY \\ Departamento de Análisis Matemático y Matemática Aplicada, Facultad de Matemáticas \\ Universidad Complutense de Madrid \\ Plaza de Ciencias 3, 28040, Madrid, Spain \\ E-mail: blanca.f.besoy@ucm.es
}

\begin{abstract}
Let $\left(A_{0}, A_{1}\right)$ be a Banach couple, $\left(B_{0}, B_{1}\right)$ a quasi-Banach couple, $0<q \leq \infty$ and $T$ a linear operator. We prove that if $T: A_{0} \rightarrow B_{0}$ is bounded and $T: A_{1} \rightarrow B_{1}$ is compact, then the interpolated operator by the logarithmic method $T:\left(A_{0}, A_{1}\right)_{1, q, \mathbb{A}} \rightarrow\left(B_{0}, B_{1}\right)_{1, q, \mathbb{A}}$ is compact too. This result allows the extension of some limit variants of Krasnosel'skiir's compact interpolation theorem.
\end{abstract}

1. Introduction. In 1960, Krasnosel'skil [20] gave a reinforced version of the RieszThorin theorem involving compactness. He proved that if $T$ is a linear operator such that $T: L_{p_{0}} \rightarrow L_{q_{0}}$ compactly and $T: L_{p_{1}} \rightarrow L_{q_{1}}$ boundedly with $1 \leq p_{0}, p_{1}, q_{1} \leq \infty$, $1 \leq q_{0}<\infty, 0<\theta<1,1 / p=(1-\theta) / p_{0}+\theta / p_{1}$ and $1 / q=(1-\theta) / q_{0}+\theta / q_{1}$, then $T: L_{p} \rightarrow L_{q}$ is also compact. This result promoted the study of compact operators between abstract interpolation spaces. The first results were due to Lions and Peetre [21] and to Persson [23] (see also [2, 24] and the references given there). In 1992, it was proven by Cwikel [15] and Cobos, Kühn and Schonbek [12] that if $\left(A_{0}, A_{1}\right),\left(B_{0}, B_{1}\right)$ are Banach couples and $T$ is a linear operator such that $T: A_{j} \rightarrow B_{j}$ is bounded, for $j=0,1$, and one of the restrictions is compact, then the interpolated operator by the real method $T:\left(A_{0}, A_{1}\right)_{\theta, q} \rightarrow\left(B_{0}, B_{1}\right)_{\theta, q}$ is also compact. In 1998, Cobos and Persson proved in [13] that the previous result is still valid for quasi-Banach couples. As a particular application of this result, they gave an extension of Krasnosel'skiľ's theorem to Lorentz spaces with no restrictions on parameters $q_{j}$, that is to say, $0<q_{0} \neq q_{1} \leq \infty$.

2010 Mathematics Subject Classification: Primary 46M35, 47B07; Secondary 46B70, 46E30.

Key words and phrases: logarithmic interpolation methods, compact operators, LorentzZygmund spaces.

The paper is in final form and no version of it will be published elsewhere. 
The logarithmic perturbations $\left(A_{0}, A_{1}\right)_{\theta, q, \mathbb{A}}$ of the real method have attracted considerable attention in the last years (see [18, 19, 14, 3]). When $\theta=0$ and $\theta=1$, these spaces are related to the limiting interpolation spaces [5, 10, 11. Applying the logarithmic methods to the couple $\left(L_{r}, L_{\infty}\right)$ one can get generalized Lorentz-Zygmund spaces $L_{p, q, \mathbb{A}}($ see [16, 22]).

Edmunds and Opic established in [17] the following limit version of Krasnosel'skiř's theorem: let $(R, \mu)$ and $(S, \nu)$ be finite measure spaces, $1<p_{0}<p_{1} \leq \infty, 1<q_{0}<$ $q_{1} \leq \infty, 1 \leq q<\infty$ and $\alpha+1 / q>0$. If $T$ is a linear operator such that $T: L_{p_{0}}(R) \rightarrow$ $L_{q_{0}}(S)$ compactly and $T: L_{p_{1}}(R) \rightarrow L_{q_{1}}(S)$ boundedly then $T: L_{p_{0}, q, \alpha+1 / \min \left(p_{0}, q\right)}(R) \rightarrow$ $L_{q_{0}, q, \alpha+1 / \max \left(q_{0}, q\right)}(S)$ is also compact.

Later Cobos, Fernández Cabrera and Martínez [7] and Cobos and Segurado [14] obtained abstract versions of this result. They work with logarithmic interpolation methods with limit values of $\theta$ applied to Banach couples and $1 \leq q \leq \infty$. In particular, it is shown in [14] that the result of Edmunds and Opic also holds when the spaces are defined over any $\sigma$-finite measure spaces.

The first objective of this paper is to extend the abstract results for $0<q \leq \infty$ and a quasi-Banach target couple. Then, as a consequence, we prove an extended version of the limit Krasnosel'skil type result for $0<q_{0}<q_{1} \leq \infty$ and $0<q<\infty$.

The organization of the paper is as follows. In Section 2 we review the definition and some properties of limit logarithmic interpolation spaces. In Section 3 we prove the abstract compactness theorem for logarithmic spaces. As the proof is quite technical, we settle several auxiliary lemmas in advance. Finally, in Section 4 we derive the Krasnosel'skiǐ's type result.

2. Logarithmic interpolation spaces. Let $\bar{A}=\left(A_{0}, A_{1}\right)$ be a quasi-Banach couple, that is to say, two quasi-Banach spaces $A_{j}, j=0,1$, which are continuously embedded in some Hausdorff topological vector space. We put $c_{A_{j}} \geq 1$ for the constants in the quasitriangle inequality, $j=0,1$. Let $t>0$, the Peetre's $K$ - and J-functionals are defined by

$$
K(t, a)=K\left(t, a ; A_{0}, A_{1}\right)=\inf \left\{\left\|a_{0}\right\|_{A_{0}}+t\left\|a_{1}\right\|_{A_{1}}: a=a_{0}+a_{1}, a_{j} \in A_{j}, j=0,1\right\}
$$

where $a \in A_{0}+A_{1}$, and

$$
J(t, a)=J\left(t, a ; A_{0}, A_{1}\right)=\max \left\{\|a\|_{A_{0}}, t\|a\|_{A_{1}}\right\}, a \in A_{0} \cap A_{1} .
$$

Observe that $K(1, \cdot)$ is the quasi-norm of $A_{0}+A_{1}$ and $J(1, \cdot)$ the quasi-norm of $A_{0} \cap A_{1}$. In both cases, the quasi-triangular inequality holds with constant $c=\max \left\{c_{A_{0}}, c_{A_{1}}\right\}$. When $c_{A_{0}}=c_{A_{1}}=1$ we say that $\bar{A}=\left(A_{0}, A_{1}\right)$ is a Banach couple.

For a quasi-Banach couple $\bar{A}=\left(A_{0}, A_{1}\right)$, the Gagliardo completion $A_{j}^{\sim}$ of $A_{j}$ is formed of all $a \in A_{0}+A_{1}$ such that

$$
\|a\|_{A_{j}^{\sim}}:=\sup \left\{t^{-j} K(t, a): t>0\right\}<\infty,
$$

(see [1, 2, 4]). Clearly $A_{j} \hookrightarrow A_{j}^{\sim}$, where $\hookrightarrow$ means continuous embedding. Note that

$$
K\left(t, a ; A_{0}^{\sim}, A_{1}^{\sim}\right) \leq K\left(t, a ; A_{0}, A_{1}\right) \leq \max \left\{c_{A_{0}}, c_{A_{1}}\right\} K\left(t, a ; A_{0}^{\sim}, A_{1}^{\sim}\right),
$$


for $t>0$ and $a \in A_{0}+A_{1}$. Indeed, for any decomposition $a=a_{0}+a_{1}$, with $a_{j} \in A_{j} \hookrightarrow A_{j}^{\sim}$, we have

$$
K\left(t, a ; A_{0}^{\sim}, A_{1}^{\sim}\right) \leq\left\|a_{0}\right\|_{A_{0}^{\sim}}+t\left\|a_{1}\right\|_{A_{1}^{\sim}} \leq\left\|a_{0}\right\|_{A_{0}}+t\left\|a_{1}\right\|_{A_{1}} .
$$

Hence $K\left(t, a ; A_{0}^{\sim}, A_{1}^{\sim}\right) \leq K\left(t, a ; A_{0}, A_{1}\right)$. On the other hand, if $a=b_{0}+b_{1}$ with $b_{j} \in A_{j}^{\sim} \hookrightarrow A_{0}+A_{1}$, then

$$
\begin{aligned}
K\left(t, a ; A_{0}, A_{1}\right) & \leq \max \left\{c_{A_{0}}, c_{A_{1}}\right\}\left(K\left(t, b_{0} ; A_{0}, A_{1}\right)+K\left(t, b_{1} ; A_{0}, A_{1}\right)\right) \\
& \leq \max \left\{c_{A_{0}}, c_{A_{1}}\right\}\left(\left\|b_{0}\right\|_{A_{0}^{\sim}}+t\left\|b_{1}\right\|_{A_{1}}\right) .
\end{aligned}
$$

Thus $K\left(t, a ; A_{0}, A_{1}\right) \leq \max \left\{c_{A_{0}}, c_{A_{1}}\right\} K\left(t, a ; A_{0}^{\sim}, A_{1}^{\sim}\right)$. In particular, if $\bar{A}=\left(A_{0}, A_{1}\right)$ is a Banach couple, we get an equality in (1) as it can be seen in [1, Theorem V.1.5].

Let $\ell(t)=1+|\log t|, \ell \ell(t)=1+(\log (1+|\log t|))$ and for $\mathbb{A}=\left(\alpha_{0}, \alpha_{\infty}\right) \in \mathbb{R}^{2}$

$$
\ell^{\mathbb{A}}(t)=\ell^{\left(\alpha_{0}, \alpha_{\infty}\right)}(t)= \begin{cases}\ell^{\alpha_{0}}(t) & \text { if } 0<t \leq 1, \\ \ell^{\alpha_{\infty}}(t) & \text { if } 1<t<\infty,\end{cases}
$$

and define $\ell \ell^{\mathbb{A}}(t)$ similarly.

Given $0 \leq \theta \leq 1,0<q \leq \infty, \mathbb{A} \in \mathbb{R}^{2}$ and a quasi-Banach couple $\bar{A}=\left(A_{0}, A_{1}\right)$, the logarithmic interpolation space $\left(A_{0}, A_{1}\right)_{\theta, q, \mathbb{A}}$ consists of all $a \in A_{0}+A_{1}$ such that

$$
\|a\|_{\left(A_{0}, A_{1}\right)_{\theta, q, \mathbb{A}}}=\left\|\left(K\left(2^{m}, a\right) 2^{-m \theta} \ell^{\mathbb{A}}\left(2^{m}\right)\right)_{m \in \mathbb{Z}}\right\|_{\ell_{q}}<\infty .
$$

Since this definition requires the weighted sequence space $\ell_{q}\left(2^{-m \theta} \ell^{\mathbb{A}}\left(2^{m}\right)\right)$, we also use the notation $\left(A_{0}, A_{1}\right)_{\ell_{q}\left(2^{-m \theta} \ell^{\mathbb{A}}\left(2^{m}\right)\right)}$. It is not difficult to check that the quasi-norm of $\left(A_{0}, A_{1}\right)_{\theta, q, \mathbb{A}}$ is equivalent to the continuous quasi-norm

$$
\|a\|_{\left(A_{0}, A_{1}\right)_{\theta, q, \mathbb{A}}} \sim \begin{cases}\left(\int_{0}^{\infty}\left[t^{-\theta} \ell^{\mathbb{A}}(t) K(t, a)\right]^{q} \frac{d t}{t}\right)^{1 / q} & \text { if } 0<q<\infty, \\ \sup \left\{t^{-\theta} \ell^{\mathbb{A}}(t) K(t, a): t>0\right\} & \text { if } q=\infty\end{cases}
$$

See [18, 19] for more details on $\left(A_{0}, A_{1}\right)_{\theta, q, \mathbb{A}}$.

We are interested in the limiting interpolation spaces that appear when $\theta=0$ and $\theta=1$. Note that $K\left(t, a ; A_{0}, A_{1}\right)=t K\left(t^{-1}, a ; A_{1}, A_{0}\right)$ and therefore

$$
\left(A_{0}, A_{1}\right)_{\theta, q,\left(\alpha_{0}, \alpha_{\infty}\right)}=\left(A_{1}, A_{0}\right)_{1-\theta, q,\left(\alpha_{\infty}, \alpha_{0}\right)}
$$

with equal quasi-norms. In particular, $\left(A_{0}, A_{1}\right)_{0, q,\left(\alpha_{0}, \alpha_{\infty}\right)}=\left(A_{1}, A_{0}\right)_{1, q,\left(\alpha_{\infty}, \alpha_{0}\right)}$. Subsequently we focus on the case $\theta=1$.

Under the assumptions

$$
\begin{cases}\alpha_{0}+\frac{1}{q}<0 & \text { if } 0<q<\infty, \\ \alpha_{0}<0 & \text { if } q=\infty\end{cases}
$$

we see that $A_{0} \cap A_{1} \hookrightarrow\left(A_{0}, A_{1}\right)_{1, q, \mathbb{A}} \hookrightarrow A_{0}+A_{1}$, for any quasi-Banach couple $\bar{A}=\left(A_{0}, A_{1}\right)$ (see [19, Theorem 2.2]).

When $\bar{A}=\left(A_{0}, A_{1}\right)$ is a Banach couple, it will be useful to represent the space $\left(A_{0}, A_{1}\right)_{1, q, \mathbb{A}}$ by means of the J-functional. 
Let $\bar{A}=\left(A_{0}, A_{1}\right)$ be a Banach couple, $0<q \leq \infty, \mathbb{A}=\left(\alpha_{0}, \alpha_{\infty}\right) \in \mathbb{R}^{2}$ and $\mathbb{B}=$ $\left(\beta_{0}, \beta_{\infty}\right) \in \mathbb{R}^{2}$. Assume that

$$
\begin{cases}\alpha_{\infty}>0, \text { or } \alpha_{\infty}=0 \text { and } \beta_{\infty} \geq 0 & \text { if } 0<q \leq 1 \\ \alpha_{\infty}-\frac{1}{q^{\prime}}>0, \text { or } \alpha_{\infty}=\frac{1}{q^{\prime}} \text { and } \beta_{\infty}-\frac{1}{q^{\prime}}>0 & \text { if } 1<q \leq \infty\end{cases}
$$

where $1 / q+1 / q^{\prime}=1$. The space $\left(A_{0}, A_{1}\right)_{1, q, \mathbb{A}, \mathbb{B}}^{J}=\left(A_{0}, A_{1}\right)_{\ell_{q}\left(2^{-m} \ell^{\mathbb{A}}\left(2^{m}\right) \ell \ell^{\mathbb{B}}\left(2^{m}\right)\right)}^{J}$ is formed of all those $a \in A_{0}+A_{1}$ for which there exists $\left(u_{m}\right) \subseteq A_{0} \cap A_{1}$ such that

$$
\left.a=\sum_{m=-\infty}^{\infty} u_{m} \quad \text { (convergence in } A_{0}+A_{1}\right)
$$

and

$$
\left\|\left(J\left(2^{m}, u_{m}\right) 2^{-m} \ell^{\mathbb{A}}\left(2^{m}\right) \ell \ell^{\mathbb{B}}\left(2^{m}\right)\right)_{m \in \mathbb{Z}}\right\|_{\ell_{q}}<\infty .
$$

We set

$$
\|a\|_{\left(A_{0}, A_{1}\right)_{1, q, \mathbb{A}, \mathbb{B}}^{J}}=\inf \left\{\left\|\left(J\left(2^{m}, u_{m}\right) 2^{-m} \ell^{\mathbb{A}}\left(2^{m}\right) \ell \ell^{\mathbb{B}}\left(2^{m}\right)\right)\right\|_{\ell_{q}}: a=\sum_{m=-\infty}^{\infty} u_{m}\right\} .
$$

If $\mathbb{B}=(0,0)$, we simply write $\left(A_{0}, A_{1}\right)_{1, q, \mathbb{A}}^{J}$. It is proven in [3, Section 2] that under the assumptions in (4), $A_{0} \cap A_{1} \hookrightarrow\left(A_{0}, A_{1}\right)_{1, q, \mathbb{A}, \mathbb{B}} \hookrightarrow A_{0}+A_{1}$ for every Banach couple $\bar{A}=\left(A_{0}, A_{1}\right)$. If $1 \leq q \leq \infty$ there exists an equivalent continuous representation for the J-spaces (see [14, Definition 3.1]).

Let $\bar{A}=\left(A_{0}, A_{1}\right)$ be a Banach couple. If $1 \leq q \leq \infty$ and $\mathbb{A}=\left(\alpha_{0}, \alpha_{\infty}\right)$ satisfies (3), then [14, Theorems 3.5 and 3.6] state that

$$
\left(A_{0}, A_{1}\right)_{1, q, \mathbb{A}}= \begin{cases}\left(A_{0}, A_{1}\right)_{1, q, \mathbb{A}+1}^{J} & \text { if } \alpha_{\infty}+1 / q>0 \\ \left(A_{0}, A_{1}\right)_{1, q, \mathbb{A}+1,(0,1)}^{J} & \text { if } \alpha_{\infty}+1 / q=0\end{cases}
$$

with equivalent norms. Here $\mathbb{A}+\lambda=\left(\alpha_{0}+\lambda, \alpha_{\infty}+\lambda\right)$, for any $\lambda \in \mathbb{R}$. If $0<q<1$ and $\mathbb{A}=\left(\alpha_{0}, \alpha_{\infty}\right)$ satisfies [3], then [3, Theorem 3.2] shows that

$$
\left(A_{0}, A_{1}\right)_{1, q, \mathbb{A}}= \begin{cases}\left(A_{0}^{\sim}, A_{1}^{\sim}\right)_{1, q, \mathbb{A}+1 / q}^{J} & \text { if } \alpha_{\infty}+1 / q>0 \\ \left(A_{0}^{\sim}, A_{1}^{\sim}\right)_{1, q, \mathbb{A}+1 / q,(0,1 / q)}^{J} & \text { if } \alpha_{\infty}+1 / q=0\end{cases}
$$

with equivalent quasi-norms. In general, when $\alpha_{\infty}+1 / q<0$ and $0<q \leq \infty$, or $\alpha_{\infty}=0$ and $q=\infty$, the K-space $\left(A_{0}, A_{1}\right)_{1, q, \mathbb{A}}$ does not admit a J-representation (see [14, Proposition 3.4] and [3, Example 2.1]). In this case, the following result is useful. For a given quasi-Banach couple $\bar{A}=\left(A_{0}, A_{1}\right), \mathbb{A}=\left(\alpha_{0}, \alpha_{\infty}\right) \in \mathbb{R}^{2}$ and $0<q \leq \infty$ satisfying

$$
\begin{cases}\alpha_{0}+1 / q<0 \text { and } \alpha_{\infty}+1 / q<0 & \text { if } 0<q<\infty, \\ \alpha_{0}<0 \text { and } \alpha_{\infty} \leq 0 & \text { if } q=\infty\end{cases}
$$

we see that for any $\alpha>-1 / q$

$$
\left(A_{0}, A_{1}\right)_{1, q, \mathbb{A}}=\left(A_{0}+A_{1}, A_{1}\right)_{1, q,\left(\alpha_{0}, \alpha\right)},
$$

with equivalent quasi-norms. This result was proven in [14, Corollary 2.5] for Banach couples and $1 \leq q \leq \infty$, but the proof remains valid for quasi-Banach couples and $0<q \leq \infty$ by just taking into account the constant in the quasi-triangle inequality. 
3. Compactness theorem. In what follows, if $X$ and $Y$ are quantities depending on certain parameters, we write $X \lesssim Y$ if $X \leq C Y$ with a constant $C$ independent of all the parameters. We put $X \sim Y$ if $X \lesssim Y$ and $Y \lesssim X$.

Let $A$ be a quasi-Banach space. For $M>0$, we put $M U_{A}=\left\{a \in A:\|a\|_{A} \leq M\right\}$ and just $U_{A}$ when $M=1$. If $B$ is another quasi-Banach space, let $\mathcal{L}(A, B)$ denote the set of bounded linear operators from $A$ to $B$, and $\mathcal{K}(A, B)$ the set of linear compact operators from $A$ to $B$. If $\bar{A}=\left(A_{0}, A_{1}\right)$ and $\bar{B}=\left(B_{0}, B_{1}\right)$ are two quasi-Banach couples, we put $T \in \mathcal{L}(\bar{A}, \bar{B})$ if $T \in \mathcal{L}\left(A_{0}+A_{1}, B_{0}+B_{1}\right)$ and the restrictions $T: A_{j} \rightarrow B_{j}$ are also bounded with quasi-norm $\|T\|_{j}$, for $j=0,1$. If $A_{0}=A_{1}=A$ or $B_{0}=B_{1}=B$, then we simply write $T \in \mathcal{L}(A, \bar{B})$ or $T \in \mathcal{L}(\bar{A}, B)$. For $\lambda \in \mathbb{R}$, we set $\lambda^{+}=\max \{0, \lambda\}$.

Let $\bar{A}=\left(A_{0}, A_{1}\right)$ and $\bar{B}=\left(B_{0}, B_{1}\right)$ be quasi-Banach couples, $0<q \leq \infty$ and $\mathbb{A}=\left(\alpha_{0}, \alpha_{\infty}\right) \in \mathbb{R}^{2}$ satisfying $(3)$. If $T \in \mathcal{L}(\bar{A}, \bar{B})$, then $T \in \mathcal{L}\left(\bar{A}_{1, q, \mathbb{A}} ; \bar{B}_{1, q, \mathbb{A}}\right)$ and the following norm estimate holds

$$
\|T\|_{\bar{A}_{1, q, \mathbb{A}} ; \bar{B}_{1, q, \mathbb{A}}} \lesssim \begin{cases}\|T\|_{1}\left(1+\left(\log \frac{\|T\|_{0}}{\|T\|_{1}}\right)^{+}\right)^{\alpha_{\infty}^{+}-\alpha_{0}} & \text { if }\|T\|_{j} \neq 0, j=0,1 ; \\ \|T\|_{1} & \text { if }\|T\|_{j}=0, j=0 \text { or } j=1 .\end{cases}
$$

This result was proven in [8, Theorem 2.2] for Banach couples and $1 \leq q \leq \infty$. The proof remains true in our hypothesis.

Our goal in this section is to prove the compactness of the interpolated operator $T:\left(A_{0}, A_{1}\right)_{1, q, \mathbb{A}} \rightarrow\left(B_{0}, B_{1}\right)_{1, q, \mathbb{A}}$, for $\bar{A}$ a Banach couple and $\bar{B}$ a quasi-Banach couple, under the assumptions that $T: A_{1} \rightarrow B_{1}$ is compact and $T: A_{0} \rightarrow B_{0}$ is bounded. For this purpose we establish first a simplified version of this result and some auxiliary lemmas.

Lemma 3.1. Let $\bar{A}=\left(A_{0}, A_{1}\right)$ be a quasi-Banach couple and let $B$ be a quasi-Banach space. Take $\mathbb{A}=\left(\alpha_{0}, \alpha_{\infty}\right) \in \mathbb{R}^{2}$ and $0<q \leq \infty$ satisfying 3 .

1. If $T \in \mathcal{L}(B, \bar{A})$ with $T: B \rightarrow A_{1}$ compact, then $T: B \rightarrow\left(A_{0}, A_{1}\right)_{1, q, \mathbb{A}}$ is compact.

2. If $T \in \mathcal{L}(\bar{A}, B)$ with $T: A_{1} \rightarrow B$ compact, then $T:\left(A_{0}, A_{1}\right)_{1, q, \mathbb{A}} \rightarrow B$ is compact.

Proof. For the first case, the proof given in [14, Lemma 4.1 (a)] is still valid. However, for the second case, [14, Lemma 4.2 (b)] uses Hahn-Banach theorem and we have to proceed differently. It is clear that for any $m \in \mathbb{Z}$

$$
\sup \left\{\frac{K\left(2^{m}, a\right)}{\|a\|_{\bar{A}_{1, q, \mathbb{A}}}}: a \in \bar{A}_{1, q, \mathbb{A}}, a \neq 0\right\} \leq 2^{m} \ell^{-\mathbb{A}}\left(2^{m}\right) .
$$

Given $\varepsilon>0$, we fix $m<0$ such that $2^{m} \ell^{-\mathbb{A}}\left(2^{m}\right) \leq \varepsilon /\left(4 c_{B}\|T\|_{A_{0}, B}\right)$. Using $[9]$, we see that for any $a \in U_{\bar{A}_{1, q, \mathbb{A}}}$ there exists $a_{j} \in A_{j}, j=0,1$, such that $a=a_{0}+a_{1}$ and

$$
\left\|a_{0}\right\|_{A_{0}}+2^{m}\left\|a_{1}\right\|_{A_{1}} \leq 2 K\left(2^{m}, a\right) \leq 2^{m+1} \ell^{-\mathbb{A}}\left(2^{m}\right) \leq \varepsilon /\left(2 c_{B}\|T\|_{A_{0}, B}\right) .
$$

Let $M=2^{-m} \varepsilon /\left(2 c_{B}\|T\|_{A_{0}, B}\right)$. By compactness of the operator $T: A_{1} \rightarrow B$, there exists $\left\{b_{1}, \ldots, b_{k}\right\} \subset B$ such that $\min \left\{\left\|T x-b_{j}\right\|_{B}: 1 \leq j \leq k\right\} \leq \varepsilon /\left(2 c_{B}\right)$, for every $x \in M U_{A_{1}}$. Consequently, for each $a \in U_{\bar{A}_{1, q, \mathbb{A}}}$ we can take $j \in\{1, \ldots, k\}$ such that $\left\|T a_{1}-b_{j}\right\|_{B} \leq \varepsilon /\left(2 c_{B}\right)$ and

$$
\left\|T a-b_{j}\right\|_{B} \leq c_{B}\left(\left\|T a_{0}\right\|_{B}+\left\|T a_{1}-b_{j}\right\|_{B}\right) \leq \varepsilon \text {. }
$$

Therefore, $T:\left(A_{0}, A_{1}\right)_{1, q, \mathbb{A}} \rightarrow B$ is compact. 
Lemma 3.2. Let $\bar{A}=\left(A_{0}, A_{1}\right)$ be a Banach couple. Let $\bar{B}=\left(B_{0}, B_{1}\right)$ be a quasi-Banach couple and $T \in \mathcal{L}(\bar{A}, \bar{B})$. If $T: A_{1} \rightarrow B_{1}$ is compact, then $T: A_{1}^{\sim} \rightarrow B_{1}^{\sim}$ is also compact. Proof. Let $\varepsilon>0$ and $a \in U_{A_{1}^{\sim}}=\left\{a \in A_{0}+A_{1}: \sup _{t>0} K(t, a) / t \leq 1\right\}$. For every $n \in \mathbb{N}$ there exist $a_{0 n} \in A_{0}$ and $a_{1 n} \in A_{1}$ such that $a=a_{0 n}+a_{1 n}$ and $\left\|a_{0 n}\right\|_{A_{0}}+1 / n\left\|a_{1 n}\right\|_{A_{1}} \leq$ $2 K(1 / n, a) \leq 2 / n$. Note that $\lim _{n \rightarrow \infty} T a_{1 n}=T a$ in $B_{0}+B_{1}$, since $\lim _{n \rightarrow \infty} a_{1 n}=a$ in $A_{0}+A_{1}$. Moreover, the sequence $\left(a_{1 n}\right)$ is contained in $2 U_{A_{1}}$ and the operator $T$ is compact from $A_{1}$ to $B_{1}$, therefore there exists a subsequence $\left(T a_{1 n^{\prime}}\right)$ that is convergent in $B_{1}$. Using compatibility, we deduce that $T a_{1 n^{\prime}} \stackrel{n^{\prime} \rightarrow \infty}{\longrightarrow} T a$ in $B_{1}$ and then we can find $n_{0}^{\prime} \in \mathbb{N}$ such that $\left\|T a_{1 n_{0}^{\prime}}-T a\right\|_{B_{1}} \leq \varepsilon /\left(2 c_{B_{1}}\right)$.

Again by compactness of $T: A_{1} \rightarrow B_{1}$, there exists $\left\{b_{1}, \ldots, b_{k}\right\} \subset B_{1}$ such that $\min \left\{\left\|T x-b_{j}\right\|_{B_{1}}: 1 \leq j \leq k\right\} \leq \varepsilon /\left(2 c_{B_{1}}\right)$, for every $x \in 2 U_{A_{1}}$. Hence, we can take $j \in\{1, \ldots, k\}$ such that $\left\|T a_{1 n_{0}^{\prime}}-b_{j}\right\|_{B_{1}} \leq \varepsilon /\left(2 c_{B_{1}}\right)$ and

$\left\|T a-b_{j}\right\|_{B_{1}} \leq c_{B_{1}}\left(\left\|T a-T a_{1 n_{0}^{\prime}}\right\|_{B_{1}}+\left\|T a_{1 n_{0}^{\prime}}-b_{j}\right\|_{B_{1}}\right) \leq c_{B_{1}}\left(\varepsilon /\left(2 c_{B_{1}}\right)+\varepsilon /\left(2 c_{B_{1}}\right)\right)=\varepsilon$.

Thus $T: A_{1}^{\sim} \rightarrow B_{1}$ is compact. Since $B_{1} \hookrightarrow B_{1}^{\sim}$, it follows that $T: A_{1}^{\sim} \rightarrow B_{1}^{\sim}$ is also compact.

The previous lemma for Banach couples and compactness on the restriction $T: A_{0} \rightarrow B_{0}$ was given in [7, Theorem 2.2]. The formulation of the next two lemmas corresponds to [6. Lemma 2.3 and Corollary 2.2] in the Banach case. The proofs can be found in [9. Lemma 3.2 and Lemma 3.3] for quasi-Banach spaces and bilinear operators.

Lemma 3.3. Let $A, B, Z$ be quasi-Banach spaces, $D$ a dense subspace of $A$ and $T \in \mathcal{K}(A, B)$. Let $\left(S_{n}\right)_{n \in \mathbb{N}} \subset \mathcal{L}(B, Z)$ such that $M:=\sup \left\{\left\|S_{n}\right\|_{B, Z}: n \geq 1\right\}<\infty$. If $\lim _{n \rightarrow \infty}\left\|S_{n} T u\right\|_{Z}=0$ for all $u \in D$ then $\lim _{n \rightarrow \infty}\left\|S_{n} T\right\|_{A, Z}=0$.

Lemma 3.4. Let $\bar{A}=\left(A_{0}, A_{1}\right)$ and $\bar{B}=\left(B_{0}, B_{1}\right)$ be quasi-Banach couples and let $A, B$ be intermediate spaces with respect to $\bar{A}$ and $\bar{B}$, respectively. Assume that $T \in$ $\mathcal{L}\left(A_{0}+A_{1}, B_{0}+B_{1}\right) \cap \mathcal{K}(A, B)$. Let $X$ be a quasi-Banach space and $\left(R_{n}\right)_{n \in \mathbb{N}} \subset \mathcal{L}(X, A)$ such that $M:=\sup \left\{\left\|R_{n}\right\|_{X, A}: n \geq 1\right\}<\infty$ and $\lim _{n \rightarrow \infty}\left\|T R_{n}\right\|_{X, B_{0}+B_{1}}=0$. Then $\lim _{n \rightarrow \infty}\left\|T R_{n}\right\|_{X, B}=0$.

Let $\left(\lambda_{m}\right)$ be a sequence of positive numbers and $\left(W_{m}\right)$ a sequence of quasi-Banach spaces with the same constant $c \geq 1$ in the quasi-triangle inequality. For any $0<q \leq \infty$, we put

$$
\ell_{q}\left(\lambda_{m} W_{m}\right)=\left\{w=\left(w_{m}\right)_{m \in \mathbb{Z}}: w_{m} \in W_{m} \text { and }\left(\lambda_{m}\left\|w_{m}\right\|_{W_{m}}\right) \in \ell_{q}\right\} .
$$

The quasi-norm in $\ell_{q}\left(\lambda_{m} W_{m}\right)$ is given by $\|w\|_{\ell_{q}\left(\lambda_{m} W_{m}\right)}=\left\|\left(\lambda_{m}\left\|w_{m}\right\|_{W_{m}}\right)_{m \in \mathbb{Z}}\right\|_{\ell_{q}}$.

Now we establish the analogous results to [14, Lemma 4.2].

Lemma 3.5. Let $\left(W_{m}\right)_{m \in \mathbb{N}}$ be a sequence of quasi-Banach spaces with constant $c \geq 1$ in the quasi-triangle inequality. Let $\mathbb{A}=\left(\alpha_{0}, \alpha_{\infty}\right) \in \mathbb{R}^{2}$ and $0<q \leq \infty$ satisfying (3). Then

$$
\left(\ell_{\infty}\left(W_{m}\right), \ell_{\infty}\left(2^{-m} W_{m}\right)\right)_{1, q, \mathbb{A}} \hookrightarrow \ell_{q}\left(2^{-m} \ell^{\mathbb{A}}\left(2^{m}\right) W_{m}\right) .
$$

Proof. Let $x=\left(x_{m}\right) \in\left(\ell_{\infty}\left(W_{m}\right), \ell_{\infty}\left(2^{-m} W_{m}\right)\right)_{1, q, \mathbb{A}}$. Given any decomposition $x=y+z$ with $y=\left(y_{m}\right) \in \ell_{\infty}\left(W_{m}\right)$ and $z=\left(z_{m}\right) \in \ell_{\infty}\left(2^{-m}\left(W_{m}\right)\right)$, we have

$$
\left\|x_{k}\right\|_{W_{k}} \leq c\left(\left\|y_{k}\right\|_{W_{k}}+\left\|z_{k}\right\|_{W_{k}}\right) \leq c\left(\|y\|_{\ell_{\infty}\left(W_{m}\right)}+2^{k}\|z\|_{\ell_{\infty}\left(2^{-m} W_{m}\right)}\right), k \in \mathbb{Z} .
$$


Then $\left\|x_{k}\right\|_{W_{k}} \leq c K\left(2^{k}, x ; \ell_{\infty}\left(W_{m}\right), \ell_{\infty}\left(2^{-m} W_{m}\right)\right)$ for every $k \in \mathbb{Z}$, which yields that $\|x\|_{\ell_{q}\left(2^{-m} \ell^{\mathbb{A}}\left(2^{m}\right) W_{m}\right)} \leq c\|x\|_{\left(\ell_{\infty}\left(W_{m}\right), \ell_{\infty}\left(2^{-m} W_{m}\right)\right)_{1, q, \mathbb{A}}}$.

For a sequence of Banach spaces we also have the following result.

LEMmA 3.6. Let $\left(W_{m}\right)_{m \in \mathbb{N}}$ be a sequence of Banach spaces. Let $\mathbb{A}=\left(\alpha_{0}, \alpha_{\infty}\right) \in \mathbb{R}^{2}$ and $0<q \leq 1$ satisfying 3 .

1. If $\alpha_{\infty}+1 / q>0$, then

2. If $\alpha_{\infty}+1 / q=0$, then

$$
\ell_{q}\left(2^{-m} \ell^{\mathbb{A}+1 / q}\left(2^{m}\right) W_{m}\right) \hookrightarrow\left(\ell_{1}\left(W_{m}\right), \ell_{1}\left(2^{-m} W_{m}\right)\right)_{1, q, \mathbb{A}} .
$$

$$
\ell_{q}\left(2^{-m} \ell^{\mathbb{A}+1 / q}\left(2^{m}\right) \ell \ell^{(0,1 / q)}\left(2^{m}\right) W_{m}\right) \hookrightarrow\left(\ell_{1}\left(W_{m}\right), \ell_{1}\left(2^{-m} W_{m}\right)\right)_{1, q, \mathbb{A}} .
$$

Proof.

1. Let $x=\left(x_{m}\right) \in \ell_{q}\left(2^{-m} \ell^{\mathbb{A}+1 / q}\left(2^{m}\right) W_{m}\right)$ and let $\delta_{m}^{k}$ the Kronecker delta. We set $u_{k}=\left(\delta_{m}^{k} x_{k}\right)_{m \in \mathbb{Z}} \in \ell_{1}\left(W_{m}\right) \cap \ell_{1}\left(2^{-m} W_{m}\right) \hookrightarrow \ell_{1}\left(W_{m}\right)^{\sim} \cap \ell_{1}\left(2^{-m} W_{m}\right)^{\sim}$. Using [6], we now derive that

$$
\begin{aligned}
\|x\|_{\left(\ell_{1}\left(W_{m}\right), \ell_{1}\left(2^{-m} W_{m}\right)\right)_{1, q, \mathbb{A}}} \sim\|x\|_{\left(\ell_{1}\left(W_{m}\right) \sim, \ell_{1}\left(2^{-m} W_{m}\right) \sim\right)_{1, q, \mathbb{A}+1 / q}^{J}} & \leq\left(\sum_{k=-\infty}^{\infty}\left[2^{-k} \ell^{\mathbb{A}+1 / q}\left(2^{k}\right) J\left(2^{k}, u_{k} ; \ell_{1}\left(W_{m}\right)^{\sim}, \ell_{1}\left(2^{-m} W_{m}\right)^{\sim}\right)\right]^{q}\right)^{1 / q} \\
& \leq\left(\sum_{k=-\infty}^{\infty}\left[2^{-k} \ell^{\mathbb{A}+1 / q}\left(2^{k}\right) J\left(2^{k}, u_{k} ; \ell_{1}\left(W_{m}\right), \ell_{1}\left(2^{-m} W_{m}\right)\right)\right]^{q}\right)^{1 / q} \\
& =\|x\|_{\ell_{q}\left(2^{-k} \ell^{\mathbb{A}+1 / q}\left(2^{k}\right)\right)} .
\end{aligned}
$$

2. This case can be handled as the previous one but using the appropriate equality of $(6)$.

We now prove the main result of this section.

THEOREM 3.7. Let $\bar{A}=\left(A_{0}, A_{1}\right)$ be a Banach couple. Let $\bar{B}=\left(B_{0}, B_{1}\right)$ be a quasiBanach couple and $T \in \mathcal{L}(\bar{A}, \bar{B})$ such that $T: A_{1} \rightarrow B_{1}$ is compact. For any $\mathbb{A}=$ $\left(\alpha_{0}, \alpha_{\infty}\right) \in \mathbb{R}^{2}$ and $0<q \leq \infty$ satisfying $(3)$,

$$
T:\left(A_{0}, A_{1}\right)_{1, q, \mathbb{A}} \rightarrow\left(B_{0}, B_{1}\right)_{1, q, \mathbb{A}}
$$

is also compact.

Proof. Step 1 . Let $0<q \leq 1$ and assume that $\alpha_{\infty}+1 / q \geq 0$. For $m \in \mathbb{Z}$, let

$$
\begin{aligned}
G_{m} & =\left(A_{0}^{\sim} \cap A_{1}^{\sim}, J\left(2^{m}, \cdot ; A_{0}^{\sim}, A_{1}^{\sim}\right)\right) \text { and } \\
F_{m} & =\left(B_{0}^{\sim}+B_{1}^{\sim}, K\left(2^{m}, \cdot ; B_{0}^{\sim}, B_{1}^{\sim}\right)\right) .
\end{aligned}
$$

We define $\mu_{m}=2^{-m} \ell^{\mathbb{A}}\left(2^{m}\right)$ and

$$
\lambda_{m}= \begin{cases}2^{-m} \ell^{\mathbb{A}+1 / q}\left(2^{m}\right) & \text { if } \alpha_{\infty}+1 / q>0, \\ 2^{-m} \ell^{\mathbb{A}+1 / q}\left(2^{m}\right) \ell \ell^{(0,1 / q)}\left(2^{m}\right) & \text { if } \alpha_{\infty}+1 / q=0 .\end{cases}
$$

By (1) and (6), we have

$$
\left(A_{0}^{\sim}, A_{1}^{\sim}\right)_{\ell_{q}\left(\mu_{m}\right)}=\left(A_{0}, A_{1}\right)_{\ell_{q}\left(\mu_{m}\right)}=\left(A_{0}^{\sim}, A_{1}^{\sim}\right)_{\ell_{q}\left(\lambda_{m}\right)}^{J}
$$

with equivalent quasi-norms. 
Consider the operators $\pi(u)=\sum_{m} u_{m}$ and $j b=(\ldots, b, b, b, \ldots)$. Observe that

$$
\pi: \ell_{q}\left(\lambda_{m} G_{m}\right) \rightarrow\left(A_{0}^{\sim}, A_{1}^{\sim}\right) \ell_{q}\left(\mu_{m}\right)
$$

is a metric surjection if we consider on $\left(A_{0}^{\sim}, A_{1}^{\sim}\right)_{\ell_{q}\left(\mu_{m}\right)}$ the J-quasi-norm. Moreover, restrictions $\pi: \ell_{1}\left(2^{m j} G_{m}\right) \rightarrow A_{j}^{\sim}, j=0,1$, are bounded operators with norm $\leq 1$. On the other hand,

$$
j:\left(B_{0}^{\sim}, B_{1}^{\sim}\right)_{1, q, \mathbb{A}} \rightarrow \ell_{q}\left(\mu_{m} F_{m}\right)
$$

is a metric injection and restrictions $j: B_{j}^{\sim} \rightarrow \ell_{\infty}\left(2^{-m j} F_{m}\right), j=0,1$, are bounded with quasi-norm $\leq 1$. Applying Lemma 3.5 and Lemma 3.6 we obtain the following diagram that illustrates the situation

\begin{tabular}{ccccccc}
$\ell_{1}\left(G_{m}\right)$ & $\stackrel{\pi}{\longrightarrow}$ & $A_{0}^{\sim}$ & $\stackrel{T}{\longrightarrow}$ & $B_{0}^{\sim}$ & $\stackrel{j}{\longrightarrow}$ & $\ell_{\infty}\left(F_{m}\right)$ \\
$\ell_{1}\left(2^{-m} G_{m}\right)$ & $\stackrel{\pi}{\longrightarrow}$ & $A_{1}^{\sim}$ & $\stackrel{T}{\longrightarrow}$ & $B_{1}^{\sim}$ & $\stackrel{j}{\longrightarrow}$ & $\ell_{\infty}\left(2^{-m} F_{m}\right)$ \\
\hline \hline$\ell_{1}\left(G_{m}\right)_{1, q, \mathbb{A}}$ & $\stackrel{\pi}{\longrightarrow}$ & $\left(A_{0}^{\sim}, A_{1}^{\sim}\right)_{1, q, \mathbb{A}}$ & $\stackrel{T}{\longrightarrow}$ & $\left(B_{0}^{\sim}, B_{1}^{\sim}\right)_{1, q, \mathbb{A}}$ & $\stackrel{j}{\longrightarrow}$ & $\bar{\ell}_{\infty}\left(F_{m}\right)_{1, q, \mathbb{A}}$, \\
$\uparrow$ & & & & & & \\
$\downarrow$ & & & & $\ell_{q}\left(\mu_{m} F_{m}\right)$
\end{tabular}

where

$$
\begin{aligned}
& \bar{\ell}_{1}\left(G_{m}\right)_{1, q, \mathbb{A}}:=\left(\ell_{1}\left(G_{m}\right), \ell_{1}\left(2^{-m} G_{m}\right)\right)_{1, q, \mathbb{A}} \text { and } \\
& \bar{\ell}_{\infty}\left(F_{m}\right)_{1, q, \mathbb{A}}:=\left(\ell_{\infty}\left(F_{m}\right), \ell_{\infty}\left(2^{-m} F_{m}\right)\right)_{1, q, \mathbb{A}} .
\end{aligned}
$$

Let $\hat{T}=j T \pi$. Properties of $\pi$ and $j$ yield that compactness of $T:\left(A_{0}, A_{1}\right)_{1, q, \mathbb{A}} \rightarrow$ $\left(B_{0}, B_{1}\right)_{1, q, \mathbb{A}}$ is equivalent to compactness of $\hat{T}: \ell_{q}\left(\lambda_{m} G_{m}\right) \rightarrow \ell_{q}\left(\mu_{m} F_{m}\right)$. Observe that by Lemma $3.2 T: A_{1}^{\sim} \rightarrow B_{1}^{\sim}$ is compact and so $\hat{T}: \ell_{1}\left(2^{-m} G_{m}\right) \rightarrow \ell_{\infty}\left(2^{-m} F_{m}\right)$ is also compact. We shall check the compactness of $\hat{T}$ with the help of the following projections. For $n \in \mathbb{N}$ we define

$$
\begin{aligned}
Q_{n}\left(u_{m}\right) & =\left(\ldots, 0,0, u_{-n}, \ldots, u_{n}, 0,0, \ldots\right), \\
Q_{n}^{+}\left(u_{m}\right) & =\left(\ldots, 0,0, u_{n+1}, u_{n+2}, \ldots\right), \\
Q_{n}^{-}\left(u_{m}\right) & =\left(\ldots, u_{-n-2}, u_{-n-1}, 0,0, \ldots\right) .
\end{aligned}
$$

The identity operator on $\ell_{1}\left(G_{m}\right)+\ell_{1}\left(2^{-m} G_{m}\right)$ can be written as $I=Q_{n}+Q_{n}^{+}+Q_{n}^{-}$. These projections have the following properties:

$$
\begin{aligned}
& \left\|Q_{n}\right\|_{E, E}=\left\|Q_{n}^{+}\right\|_{E, E}=\left\|Q_{n}^{-}\right\|_{E, E}=1 \text { for } E=\ell_{1}\left(G_{m}\right), \ell_{1}\left(2^{-m} G_{m}\right), \ell_{q}\left(\lambda_{m} G_{m}\right), \\
& \left\|Q_{n}\right\|_{\ell_{1}\left(2^{-m} G_{m}\right), \ell_{1}\left(G_{m}\right)}=\left\|Q_{n}\right\|_{\ell_{1}\left(G_{m}\right), \ell_{1}\left(2^{-m} G_{m}\right)}=2^{n}, n \geq 1, \\
& \left\|Q_{n}^{+}\right\|_{\ell_{1}\left(G_{m}\right), \ell_{1}\left(2^{-m} G_{m}\right)}=2^{-(n+1)}, n \geq 1, \\
& \left\|Q_{n}^{-}\right\|_{\ell_{1}\left(2^{-m} G_{m}\right), \ell_{1}\left(G_{m}\right)}=2^{-(n+1)}, n \geq 1 .
\end{aligned}
$$

On the couple $\left(\ell_{\infty}\left(F_{m}\right), \ell_{\infty}\left(2^{-m} F_{m}\right)\right)$ we can define similar projections $P_{n}, P_{n}^{+}, P_{n}^{-}$satisfying analogous properties.

We have

$$
\hat{T}=\hat{T} Q_{n}+\hat{T} Q_{n}^{-}+\hat{T} Q_{n}^{+}=\hat{T} Q_{n}+\hat{T} Q_{n}^{-}+P_{n} \hat{T} Q_{n}^{+}+P_{n}^{-} \hat{T} Q_{n}^{+}+P_{n}^{+} \hat{T} Q_{n}^{+} .
$$

Next we show that $\hat{T} Q_{n}, P_{n} \hat{T} Q_{n}^{+}$, and $P_{n}^{-} \hat{T} Q_{n}^{+}$are compact from $\ell_{q}\left(\lambda_{m} G_{m}\right)$ to $\ell_{q}\left(\mu_{m} F_{m}\right)$ and that the quasi-norms of the other two operators converge to 0 . 
Using (11) and Lemma 3.6, we have the factorization

$$
\ell_{q}\left(\lambda_{m} G_{m}\right) \longleftrightarrow \ell_{1}\left(G_{m}\right)+\ell_{1}\left(2^{-m} G_{m}\right) \stackrel{Q_{n}}{\longrightarrow} \ell_{1}\left(G_{m}\right) \stackrel{\hat{T}}{\longrightarrow} \ell_{\infty}\left(F_{m}\right)
$$

which allows applying Lemma 3.1 to obtain the compactness of

$$
\hat{T} Q_{n}: \ell_{q}\left(\lambda_{m} G_{m}\right) \rightarrow\left(\ell_{\infty}\left(F_{m}\right), \ell_{\infty}\left(2^{-m} F_{m}\right)\right)_{1, q, \mathbb{A}} .
$$

Now from Lemma 3.5 we conclude that $\hat{T} Q_{n}: \ell_{q}\left(\lambda_{m} G_{m}\right) \rightarrow \ell_{q}\left(\mu_{m} F_{m}\right)$ is compact.

Considering (10), (12), the analogous properties to (10) and 11 for the operator $P_{n}$ and Lemma 3.5, we have the factorization

$$
\begin{aligned}
& \ell_{1}\left(G_{m}\right) Q_{n}^{+} \\
& \ell_{1}\left(2^{-m} G_{m}\right) \stackrel{\hat{T}}{\rightarrow} \ell_{\infty}\left(2^{-m} F_{m}\right) \stackrel{P_{n}}{\rightarrow} \ell_{\infty}\left(F_{m}\right) \cap \ell_{\infty}\left(2^{-m} F_{m}\right) \hookrightarrow \ell_{q}\left(\mu_{m} F_{m}\right) . \\
& \ell_{1}\left(2^{-m} G_{m}\right) \overleftrightarrow{Q_{n}^{+}}
\end{aligned}
$$

Thus, by Lemma 3.1 and Lemma 3.6 , the operator $P_{n} \hat{T} Q_{n}^{+}: \ell_{q}\left(\lambda_{m} G_{m}\right) \rightarrow \ell_{q}\left(\mu_{m} F_{m}\right)$ is compact.

For $P_{n}^{-} \hat{T} Q_{n}^{+}$, we first use 10 and 12 to get the next diagram

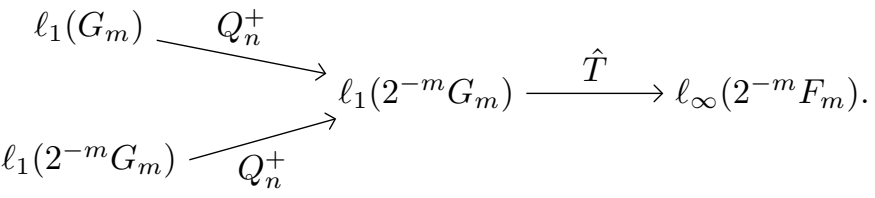

Again from Lemma 3.1 and Lemma 3.6. we infer the compactness of $T Q_{n}^{+}: \ell_{q}\left(\lambda_{m} G_{m}\right) \rightarrow$ $\ell_{\infty}\left(2^{-m} F_{m}\right)$. Now using the analogous property to 13 for the operator $P_{n}^{-}$, we have the factorization

$$
\ell_{q}\left(\lambda_{m} G_{m}\right) \stackrel{\hat{T} Q_{n}^{+}}{\longrightarrow} \ell_{\infty}\left(2^{-m} F_{m}\right) \stackrel{P_{n}^{-}}{\stackrel{P_{n}^{-}}{\longrightarrow}} \ell_{\infty}\left(2^{-m} F_{m}\right) .
$$

Applying again Lemma 3.1 and Lemma 3.5 we deduce that $P_{n}^{-} \hat{T} Q_{n}^{+}: \ell_{q}\left(\lambda_{m} G_{m}\right) \rightarrow$ $\ell_{q}\left(\mu_{m} F_{m}\right)$ is compact.

We shall now prove that $\left\|\hat{T} Q_{n}^{-}\right\|_{\ell_{q}\left(\lambda_{m} G_{m}\right), \ell_{q}\left(\mu_{m} F_{m}\right)} \stackrel{n \rightarrow \infty}{\longrightarrow} 0$. Using $\sqrt{13}$ we get

$$
\left\|\hat{T} Q_{n}^{-}\right\|_{\ell_{1}\left(2^{-m} G_{m}\right), \ell_{\infty}\left(F_{m}\right)+\ell_{\infty}\left(2^{-m} F_{m}\right)} \leq 2^{-(n+1)}\|\hat{T}\|_{\ell_{1}\left(G_{m}\right), \ell_{\infty}\left(F_{m}\right)+\ell_{\infty}\left(2^{-m} F_{m}\right)} \stackrel{n \rightarrow \infty}{\longrightarrow} 0 .
$$

Then Lemma 3.4 implies that $\left\|\hat{T} Q_{n}^{-}\right\|_{\ell_{1}\left(2^{-m} G_{m}\right), \ell_{\infty}\left(2^{-m} F_{m}\right)} \stackrel{n \rightarrow \infty}{\longrightarrow} 0$. Note also that $\left\|\hat{T} Q_{n}^{-}\right\|_{\ell_{1}\left(G_{m}\right), \ell_{\infty}\left(F_{m}\right)} \leq\|\hat{T}\|_{\ell_{1}\left(G_{m}\right), \ell_{\infty}\left(F_{m}\right)} \quad$ for every $n \in \mathbb{N}$. 
Thus, using (8), Lemma 3.5 and Lemma 3.6, we conclude that

$$
\begin{aligned}
\lim _{n \rightarrow \infty}\left\|\hat{T} Q_{n}^{-}\right\|_{\ell_{q}\left(\lambda_{m} G_{m}\right), \ell_{q}\left(\mu_{m} F_{m}\right)} & \lesssim \lim _{n \rightarrow \infty}\left\|\hat{T} Q_{n}^{-}\right\|_{\bar{\ell}_{1}\left(G_{m}\right)_{1, q, \mathbb{A}} ; \bar{\ell}_{\infty}\left(F_{m}\right)_{1, q, \mathbb{A}}} \\
& \lesssim \lim _{n \rightarrow \infty}\left\|\hat{T} Q_{n}^{-}\right\|_{1}\left(1+\left(\log \frac{\left\|\hat{T} Q_{n}^{-}\right\|_{0}}{\left\|\hat{T} Q_{n}^{-}\right\|_{1}}\right)^{+}\right)^{\alpha_{\infty}^{+}-\alpha_{0}}=0 .
\end{aligned}
$$

Now we show that $\lim _{n \rightarrow \infty}\left\|P_{n}^{+} \hat{T} Q_{n}^{+}\right\|_{\ell_{q}\left(\lambda_{m} G_{m}\right), \ell_{q}\left(\mu_{m} F_{m}\right)}=0$. We define

$$
D=\left\{u=\left(u_{m}\right)_{m=-\infty}^{\infty}: u_{m} \in G_{m} \text { with a finite number of no-null coordinates }\right\} .
$$

Since $D$ is dense in $\ell_{1}\left(2^{-m} G_{m}\right)$ and for any $u \in D$,

$$
\left\|P_{n}^{+} \hat{T} u\right\|_{\ell_{\infty}\left(2^{-m} F_{m}\right)} \leq 2^{-(n+1)}\|\hat{T}\|_{\ell_{1}\left(G_{m}\right), \ell_{\infty}\left(F_{m}\right)}\|u\|_{\ell_{1}\left(G_{m}\right)} \stackrel{n \rightarrow \infty}{\longrightarrow} 0
$$

by Lemma 3.3 we deduce that

$$
\lim _{n \rightarrow \infty}\left\|P_{n}^{+} \hat{T} Q_{n}^{+}\right\|_{\ell_{1}\left(2^{-m} G_{m}\right), \ell_{\infty}\left(2^{-m} F_{m}\right)} \leq \lim _{n \rightarrow \infty}\left\|P_{n}^{+} \hat{T}\right\|_{\ell_{1}\left(2^{-m} G_{m}\right), \ell_{\infty}\left(2^{-m} F_{m}\right)}=0 .
$$

Then, proceeding as in the previous case we infer that

$$
\lim _{n \rightarrow \infty}\left\|P_{n}^{+} \hat{T} Q_{n}^{+}\right\|_{\ell_{q}\left(\lambda_{m} G_{m}\right), \ell_{q}\left(\lambda_{m} F_{m}\right)}=0 .
$$

Step 2. Let $0<q \leq 1$ and suppose now that $\alpha_{\infty}+1 / q<0$. Take $\alpha>-1 / q$. By (7), we get $\left(A_{0}, A_{1}\right)_{1, q, \mathbb{A}}=\left(A_{0}+A_{1}, A_{1}\right)_{1, q,\left(\alpha_{0}, \alpha\right)}$ and $\left(B_{0}, B_{1}\right)_{1, q, \mathbb{A}}=\left(B_{0}+B_{1}, B_{1}\right)_{1, q,\left(\alpha_{0}, \alpha\right)}$. Applying the previous case we prove the compactness of

$$
T:\left(A_{0}, A_{1}\right)_{1, q, \mathbb{A}}=\left(A_{0}+A_{1}, A_{1}\right)_{1, q,\left(\alpha_{0}, \alpha\right)} \rightarrow\left(B_{0}+B_{1}, B_{1}\right)_{1, q,\left(\alpha_{0}, \alpha\right)}=\left(B_{0}, B_{1}\right)_{1, q, \mathbb{A}} .
$$

Step 3. Assume now that $1<q \leq \infty$. In this case we can proceed as when $0<q \leq 1$ but defining

$$
\lambda_{m}= \begin{cases}2^{-m} \ell^{\mathbb{A}+1}\left(2^{m}\right) & \text { if } \alpha_{\infty}+1 / q>0, \\ 2^{-m} \ell^{\mathbb{A}+1}\left(2^{m}\right) \ell \ell^{(0,1)}\left(2^{m}\right) & \text { if } \alpha_{\infty}+1 / q=0 \text { and } 1<q<\infty,\end{cases}
$$

and using (5) instead of [6] and [14, Lemma 4.2] instead of Lemma 3.1

This completes the proof.

The corresponding result for the $0, q, \mathbb{A}$-method is a consequence of $(2)$ and reads as follows.

Corollary 3.8. Let $\bar{A}=\left(A_{0}, A_{1}\right)$ be a Banach couple. Let $\bar{B}=\left(B_{0}, B_{1}\right)$ be a quasiBanach couple and $T \in \mathcal{L}(\bar{A}, \bar{B})$ such that $T: A_{0} \rightarrow B_{0}$ is compact. For any $\mathbb{A}=$ $\left(\alpha_{0}, \alpha_{\infty}\right) \in \mathbb{R}^{2}$ and $0<q \leq \infty$ such that

$$
\begin{cases}\alpha_{\infty}+1 / q<0 & \text { if } q<\infty \\ \alpha_{\infty}<0 & \text { if } q=\infty\end{cases}
$$

we see that $T:\left(A_{0}, A_{1}\right)_{0, q, \mathbb{A}} \rightarrow\left(B_{0}, B_{1}\right)_{0, q, \mathbb{A}}$ is also compact.

4. Applications to Lorentz-Zygmund spaces. Let $(R, \mu)$ be a $\sigma$-finite measure space. For $f$ a $\mu$-measurable function on $R$, let $f^{*}$ be the non-increasing rearrangement of $f$ defined by

$$
f^{*}(t)=\inf \{s>0: \mu(\{x \in R:|f(x)|>s\}) \leq t\}
$$


Let $0<p, q \leq \infty$ and $\mathbb{A}=\left(\alpha_{0}, \alpha_{\infty}\right) \in \mathbb{R}^{2}$. The generalized Lorentz-Zygmund space $L_{p, q, \mathbb{A}}(R, \mu)$ is formed of all the (classes of) $\mu$-measurable functions $f$ on $R$ having a finite quasi-norm

$$
\|f\|_{p, q, \mathbb{A}}=\left(\int_{0}^{\mu(R)}\left[t^{1 / p} \ell^{\mathbb{A}}(t) f^{*}(t)\right]^{q} \frac{d t}{t}\right)^{1 / q} .
$$

See [22, 16].

Now we are going to extend the result given in [14, Corollary 4.5] to the case $0<q<\infty$ and $0<q_{0}<q_{1} \leq \infty$.

THEOREM 4.1. Let $(R, \mu)$ and $(S, \nu)$ be $\sigma$-finite measure spaces. Take $1<p_{0}<p_{1} \leq \infty$, $0<q_{0}<q_{1} \leq \infty, 0<q<\infty$ and $\mathbb{A}=\left(\alpha_{0}, \alpha_{\infty}\right) \in \mathbb{R}^{2}$ with $\alpha_{\infty}+1 / q<0<\alpha_{0}+1 / q$. Let $T$ be a linear operator such that

$$
\begin{aligned}
& T: L_{p_{0}}(R) \rightarrow L_{q_{0}}(S) \text { is compact and } \\
& T: L_{p_{1}}(R) \rightarrow L_{q_{1}}(S) \text { is bounded. }
\end{aligned}
$$

Then $T: L_{p_{0}, q, \mathbb{A}+1 / \min \left(p_{0}, q\right)}(R) \rightarrow L_{q_{0}, q, \mathbb{A}+1 / \max \left(q_{0}, q\right)}(S)$ is also compact.

Proof. By Corollary 3.8

$$
T:\left(L_{p_{0}}(R), L_{p_{1}}(R)\right)_{0, q, \mathbb{A}} \rightarrow\left(L_{q_{0}}(S), L_{q_{1}}(S)\right)_{0, q, \mathbb{A}}
$$

is compact. On the other hand, according to [2, Theorem 5.2.1] for any $r<q_{0}$ we have

$$
\begin{aligned}
& L_{p_{0}}(R)=\left(L_{1}(R), L_{\infty}(R)\right)_{1-1 / p_{0}, p_{0}}, \\
& L_{p_{1}}(R)=\left(L_{1}(R), L_{\infty}(R)\right)_{1-1 / p_{1}, p_{1}}, \\
& L_{q_{0}}(S)=\left(L_{r}(S), L_{\infty}(S)\right)_{1-r / q_{0}, q_{0}}, \\
& L_{q_{1}}(S)=\left(L_{r}(S), L_{\infty}(S)\right)_{1-r / q_{1}, q_{1}} .
\end{aligned}
$$

It follows from [18, Theorem 4.7 and Theorem 5.9]

$$
\begin{aligned}
& \left(L_{1}(R), L_{\infty}(R)\right)_{1-1 / p_{0}, q, \mathbb{A}+1 / \min \left(p_{0}, q\right)} \hookrightarrow\left(L_{p_{0}}(R), L_{p_{1}}(R)\right)_{0, q, \mathbb{A}}, \\
& \left(L_{q_{0}}(S), L_{q_{1}}(S)\right)_{0, q, \mathbb{A}} \hookrightarrow\left(L_{r}(S), L_{\infty}(S)\right)_{1-r / q_{0}, q, \mathbb{A}+1 / \max \left(q, q_{0}\right)} .
\end{aligned}
$$

Besides by [18, Corollary 8.4] we have

$$
\begin{aligned}
& L_{p_{0}, q, \mathbb{A}+1 / \min \left(p_{0}, q\right)}=\left(L_{1}(R), L_{\infty}(R)\right)_{1-1 / p_{0}, q, \mathbb{A}+1 / \min \left(p_{0}, q\right)}, \\
& L_{q_{0}, q, \mathbb{A}+1 / \max \left(q_{0}, q\right)}=\left(L_{r}(S), L_{\infty}(S)\right)_{1-r / q_{0}, q, \mathbb{A}+1 / \max \left(q, q_{0}\right)} .
\end{aligned}
$$

Consequently, the operator

$$
\begin{aligned}
T: L_{p_{0}, q, \mathbb{A}+1 / \min \left(p_{0}, q\right)} \hookrightarrow\left(L_{p_{0}}(R), L_{p_{1}}(R)\right)_{0, q, \mathbb{A}} \\
\\
\rightarrow\left(L_{q_{0}}(S), L_{q_{1}}(S)\right)_{0, q, \mathbb{A}} \hookrightarrow L_{q_{0}, q, \mathbb{A}+1 / \max \left(q_{0}, q\right)}
\end{aligned}
$$

is compact.

Next we consider the case of compactness on the second restriction.

Corollary 4.2. Let $(R, \mu)$ and $(S, \nu)$ be $\sigma$-finite measure spaces. Take $1 \leq p_{0}<p_{1}<\infty$, $0<q_{0}<q_{1}<\infty, 0<q<\infty$ and $\mathbb{A}=\left(\alpha_{0}, \alpha_{\infty}\right) \in \mathbb{R}^{2}$ with $\alpha_{0}+1 / q<0<\alpha_{\infty}+1 / q$. Let $T$ be a linear operator such that

$$
T: L_{p_{0}}(R) \rightarrow L_{q_{0}}(S) \text { is bounded and } T: L_{p_{1}}(R) \rightarrow L_{q_{1}}(S) \text { is compact. }
$$

Then $T: L_{p_{1}, q, \mathbb{A}+1 / \min \left(p_{1}, q\right)}(R) \rightarrow L_{q_{1}, q, \mathbb{A}+1 / \max \left(q_{1}, q\right)}(S)$ is also compact. 
Proof. By Theorem 3.7 and $(2)$,

$$
T:\left(L_{p_{1}}(R), L_{p_{0}}(R)\right)_{0, q,\left(\alpha_{\infty}, \alpha_{0}\right)} \rightarrow\left(L_{q_{1}}(S), L_{q_{0}}(S)\right)_{0, q,\left(\alpha_{\infty}, \alpha_{0}\right)}
$$

is compact.

Using [2, Theorem 5.2.1 and Theorem 3.4.1(a)], for any $r<q_{0}$ we get

$$
\begin{aligned}
L_{p_{0}}(R) & =\left(L_{\infty}(R), L_{1}(R)\right)_{1 / p_{0}, p_{0}} \text { if } p_{0}>1, \\
L_{p_{1}}(R) & =\left(L_{\infty}(R), L_{1}(R)\right)_{1 / p_{1}, p_{1}}, \\
L_{q_{0}}(S) & =\left(L_{\infty}(S), L_{r}(S)\right)_{r / q_{0}, q_{0}}, \\
L_{q_{1}}(S) & =\left(L_{\infty}(S), L_{r}(S)\right)_{r / q_{1}, q_{1}} .
\end{aligned}
$$

It follows from [18, Theorem 4.7 and Theorem 5.9] that

$$
\begin{aligned}
& \left(L_{\infty}(R), L_{1}(R)\right)_{1 / p_{1}, q,\left(\alpha_{\infty}, \alpha_{0}\right)+1 / \min \left(p_{1}, q\right)} \hookrightarrow\left(L_{p_{1}}(R), L_{p_{0}}(R)\right)_{0, q,\left(\alpha_{\infty}, \alpha_{0}\right)} \text { and } \\
& \left(L_{q_{1}}(S), L_{q_{0}}(S)\right)_{0, q,\left(\alpha_{\infty}, \alpha_{0}\right)} \hookrightarrow\left(L_{\infty}(S), L_{r}(S)\right)_{r / q_{1}, q,\left(\alpha_{\infty}, \alpha_{0}\right)+1 / \max \left(q, q_{1}\right)} .
\end{aligned}
$$

If $p_{0}=1$, these inclusions also follow from [18, Theorem 4.7 and Theorem 5.9]. Furthermore, according to [18, Corollary 8.4] and (2) we have

$$
\begin{aligned}
L_{p_{1}, q, \mathbb{A}+1 / \min \left(p_{1}, q\right)} & =\left(L_{\infty}(R), L_{1}(R)\right)_{1 / p_{1}, q,\left(\alpha_{\infty}, \alpha_{0}\right)+1 / \min \left(p_{1}, q\right)}, \\
L_{q_{1}, q, \mathbb{A}+1 / \max \left(q_{1}, q\right)} & =\left(L_{\infty}(S), L_{r}(S)\right)_{r / q_{1}, q,\left(\alpha_{\infty}, \alpha_{0}\right)+1 / \max \left(q, q_{1}\right)} .
\end{aligned}
$$

Consequently, the operator

$$
\begin{aligned}
T: L_{p_{1}, q, \mathbb{A}+1 / \min \left(p_{1}, q\right)} \hookrightarrow\left(L_{p_{0}}(R), L_{p_{1}}(R)\right)_{1, q, \mathbb{A}} & \\
& \rightarrow\left(L_{q_{0}}(S), L_{q_{1}}(S)\right)_{1, q, \mathbb{A}} \hookrightarrow L_{q_{1}, q, \mathbb{A}+1 / \max \left(q_{1}, q\right)}
\end{aligned}
$$

is compact.

Acknowledgments. The author has been supported by FPU grant FPU16/02420 of the Spanish Ministerio de Educación, Cultura y Deporte and by MTM2017-84508-P (AEI/FEDER, UE).

\section{References}

[1] C. Bennett, R. Sharpley, Interpolation of Operators, Pure Appl. Math. 129, Academic Press, Boston, 1988.

[2] J. Bergh, J. Löfström, Interpolation Spaces. An Introduction, Grundlehren Math. Wiss. 223, Springer, Berlin, 1976.

[3] B. F. Besoy, F. Cobos, Duality for logarithmic interpolation spaces when $0<q<1$ and applications, J. Math. Anal. Appl. 466 (2018), 373-399.

[4] Y. Brudnyı̆, N. Krugljak, Interpolation Functors and Interpolation Spaces, Vol. 1, NorthHolland Math. Library 47, North-Holland, Amsterdam, 1991.

[5] F. Cobos, L. M. Fernández-Cabrera, T. Kühn, T. Ullrich, On an extreme class of real interpolation spaces, J. Funct. Anal. 256 (2009), 2321-2366.

[6] F. Cobos, L. M. Fernández-Cabrera, A. Martínez, Complex interpolation, minimal methods and compact operators, Math. Nachr. 263/264 (2004), 67-82.

[7] F. Cobos, L. M. Fernández-Cabrera, A. Martínez, On a paper of Edmunds and Opic on limiting interpolation of compact operators between $L_{p}$ spaces, Math. Nachr. 288 (2015), $167-175$. 
[8] F. Cobos, L. M. Fernández-Cabrera, A. Martínez, Estimates for the spectrum on logarithmic interpolation spaces, J. Math. Anal. Appl. 437 (2016), 292-309.

[9] F. Cobos, L. M. Fernández-Cabrera, A. Martínez, Interpolation of compact bilinear operators among quasi-Banach spaces and applications, Math. Nachr. 291 (2018), 2168-2187.

[10] F. Cobos, L. M. Fernández-Cabrera, M. Mastyło, Abstract limit J-spaces, J. Lond. Math. Soc. (2) 82 (2010), 501-525.

[11] F. Cobos, T. Kühn, Equivalence of $K$ - and J-methods for limiting real interpolation spaces, J. Funct. Anal. 261 (2011), 3696-3722.

[12] F. Cobos, T. Kühn, T. Schonbek, One-sided compactness results for Aronszajn-Gagliardo functors, J. Funct. Anal. 106 (1992), 274-313.

[13] F. Cobos, L. E. Persson, Real interpolation of compact operators between quasi-Banach spaces, Math. Scand. 82 (1998), 138-160.

[14] F. Cobos, A. Segurado, Description of logarithmic interpolation spaces by means of the J-functional and applications, J. Funct. Anal. 268 (2015), 2906-2945.

[15] M. Cwikel, Real and complex interpolation and extrapolation of compact operators, Duke Math. J. 65 (1992), 333-343.

[16] D. E. Edmunds, W. D. Evans, Hardy Operators, Function Spaces and Embeddings, Springer Monogr. Math., Springer, Berlin, 2004.

[17] D. E. Edmunds, B. Opic, Limiting variants of Krasnosel'skiı's compact interpolation theorem, J. Funct. Anal. 266 (2014), 3265-3285.

[18] W. D. Evans, B. Opic, Real interpolation with logarithmic functors and reiteration, Canad. J. Math. 52 (2000), 920-960.

[19] W. D. Evans, B. Opic, L. Pick, Real interpolation with logarithmic functors, J. Inequal. Appl. 7 (2002), 187-269.

[20] M. A. Krasnosel'skiř, On a theorem of M. Riesz, Dokl. Akad. Nauk SSSR 131 (1960), 246-248; English transl.: Soviet. Math. Dokl. 1 (1960), 229-231.

[21] J.-L. Lions, J. Peetre, Sur une classe d'espaces d'interpolation, Inst. Hautes Études Sci. Publ. Math. 19 (1964), 5-68.

[22] B. Opic, L. Pick, On generalized Lorentz-Zygmund spaces, Math. Inequal. Appl. 2 (1999), 391-467.

[23] A. Persson, Compact linear mappings between interpolation spaces, Ark. Mat. 5 (1964), $215-219$.

[24] H. Triebel, Interpolation Theory, Function Spaces, Differential Operators, North-Holland Math. Library 18, North-Holland, Amsterdam, 1978. 
